Microelectronic Engineering

Elsevier Editorial system(tm) for

Manuscript Draft

Manuscript Number:

Title: Dynamics of Set and Reset Processes on Resistive Switching Memories

Article Type: VSI:INFOS 2019

Section/Category: Nanoelectronic and photonic devices and their fabrication

Keywords: RRAM set and Reset Dynamics; Voltage and time dependency; Erlang function; Sigmoidal law; Energy barrier interface

Corresponding Author: Dr. SALVADOR DUENAS,

Corresponding Author's Institution: Universidad de Valladolid

First Author: SALVADOR DUENAS

Order of Authors: SALVADOR DUENAS; Helena Castán, PhD; Óscar G Ossorio, PhD Student; Héctor García, PhD

Abstract: In this work, we have characterized hafnium oxide based bipolar resistive switching memories (RRAM) have by measuring the small-signal conductance. The samples under study exhibit a continuum of intermediate states which can be accurately controlled by means of adequate sequence of the applied stimulus. The experimental results are analyzed to obtain information on the dynamics of the set and reset processes. This study reveals that ON-to-OFF (reset) and OFF-to-ON (set) processes are not symmetrical. Set transition is gradual and depends on the voltage stimulus according an Erlang function which consists on sum of $k$ independent and identically distributed mechanisms, each having an exponential distribution. In contrast, reset process is more abrupt and can be described by a sigmoidal law. Time dependencies of set and reset process at fixed voltage values are explored as well. Set process is gradual at any positive voltage, whereas reset process is characterized by a time constant which depends on the applied voltage. Experimental results are explained in terms of the formation of interfacial barrier between the top electrode and the conductive filament.

Suggested Reviewers: Ting-Chang Chang

tcchang3708@gmail.com

Expertise in the field

Mario Lanza

mlanzalsuda.edu. cn

Expertise in the field 


\section{Highlights}

- Dynamics of Set and Reset Processes on TiN/Ti/HfO $/$ /W-based RRAM devices

- Voltage and time dependency of Set and Reset process

- Set is described by an Erlang function.

- Reset follows a sigmoidal law.

\section{Graphical Abstract}
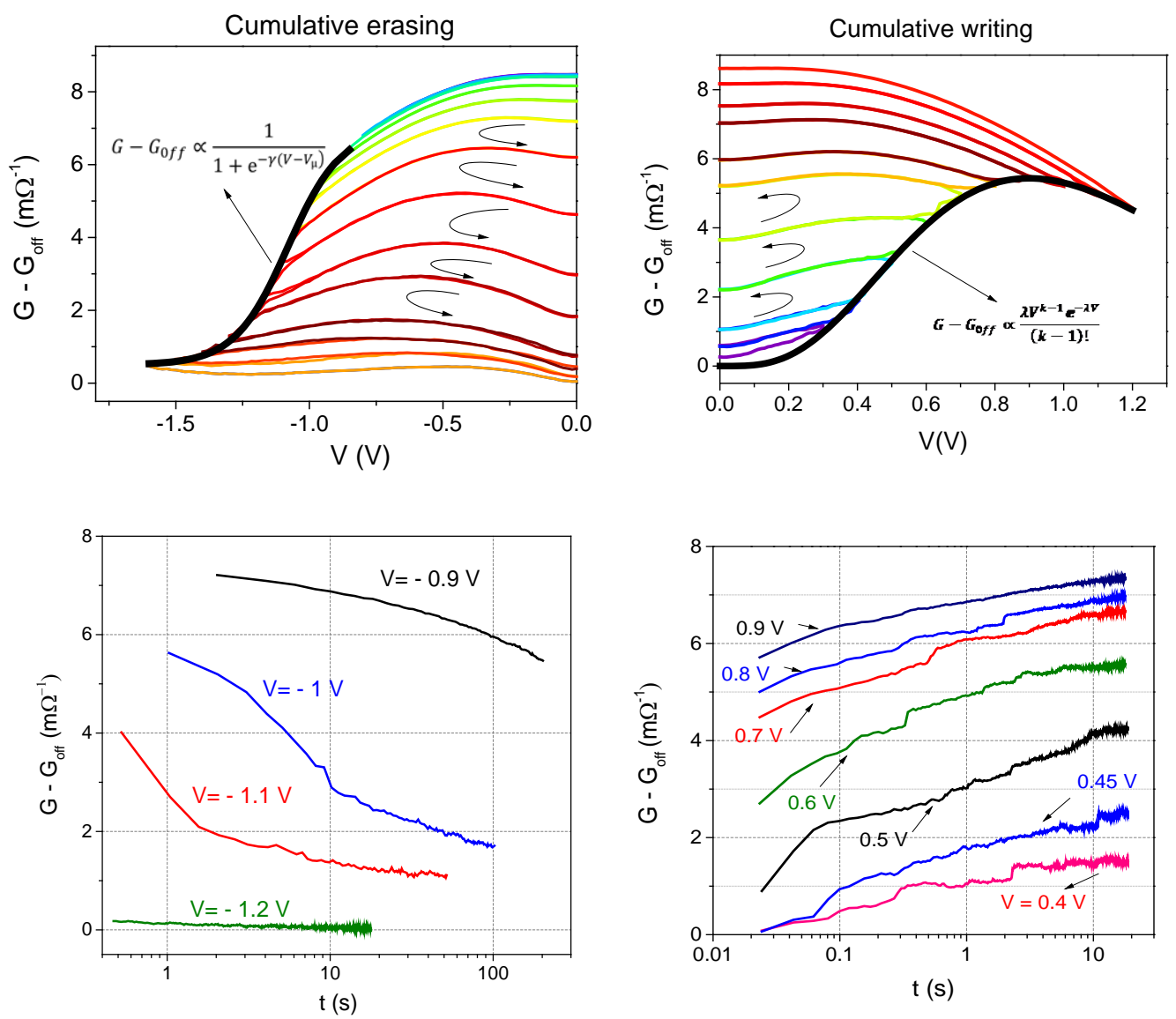


\title{
Dynamics of Set and Reset Processes on Resistive Switching Memories
}

\author{
S. Dueñas, H. Castán, O. G. Ossorio, H.García \\ University of Valladolid, Department of Electronics, Paseo de Belén 15, Valladolid, \\ Spain \\ E-mail address of corresponding author: sduenas@ele.uva.es
}

\section{Keywords}

RRAM Set and Reset Dynamics

Voltage and time dependency

Erlang function

Sigmoidal law

Energy barrier interface

\begin{abstract}
In this work, we have characterized hafnium oxide based bipolar resistive switching memories (RRAM) have by measuring the small-signal conductance. The samples under study exhibit a continuum of intermediate states which can be accurately controlled by means of adequate sequence of the applied stimulus. The experimental results are analyzed to obtain information on the dynamics of the set and reset processes. This study reveals that ON-to-OFF (reset) and OFF-to-ON (set) processes are not symmetrical. Set transition is gradual and depends on the voltage stimulus according an Erlang function which consists on sum of $\mathrm{k}$ independent and identically distributed mechanisms, each having an exponential distribution. In contrast, reset process is more abrupt and can be described by a sigmoidal law. Time dependencies of set and reset process at fixed voltage values are explored as well. Set process is gradual at any positive voltage, whereas reset process is characterized by a time constant which depends on the applied voltage. Experimental results are explained in terms of the formation of interfacial barrier between the top electrode and the conductive filament.
\end{abstract}




\section{Introduction}

Resistive random-access memories (RRAMs) have enormous potential for the development scalable and efficient non-volatile memories, but also for analog applications and brain-inspired computing systems [1]. Because of that, it is mandatory to characterize the dynamics of the switching processes as a function of the stimulus applied to the memory cells. Many works have been devoted to discussing the properties of the filaments on the low-resistance and high-resistance sates, but the dynamics and the dynamics of the set and reset transitions are more rarely investigated [2].

Hafnium oxide is one of the dielectrics more widely studied to fabricate this kind of devices. The resistive switching in $\mathrm{HfO}_{\mathrm{x}}$ is attributed to the movement of oxygen vacancies on the dielectric film, which leads to a valence change in the Hf cations and in the local conductivity [3-5]. Oxygen-deficient conductive filaments are formed during an electroforming process in which oxygen atoms are extracted from the film. Once the conductive filament is formed the device is at the low resistance or ON state (LRS). The high-resistance or OFF state (HRS) is achieved by an oxidation reaction in which oxygen vacancies are partially removed from the filaments by injecting oxygen atoms into the oxide. Subsequently, the ON state can be recovered by a new oxidation reaction in which oxygen vacancies are moved into the filament, that is, but extracting oxygen atoms by extracting oxygen atoms from the filament.

The RRAM switching dynamics has been widely studied in terms of the variation of the gradual resistance changes when applying proper voltage waveforms [6-14] like triangular voltage ramps or trains of pulses. However, few works have been dedicated to observing the capacitance changes which concurrently occurs during the resistive switching process [15-19], and the electrical properties of resistive switching memories under a.c. electrical stimulus is rarely studied in detail [20, 21].

In previous works, we have demonstrated that $\mathrm{TiN} / \mathrm{Ti} / \mathrm{HfO}_{2} / \mathrm{W}$-based resistive memories exhibit hysteretic behavior not only in the d.c. I-V characteristic but also in the small signal admittance parameters $[22,23]$. In these works, we proved that the real 
(conductance) and imaginary (capacitance) components of the admittance of RRAM memories shows well-defined minor switching loops associated with partial transitions between the ON and OFF states. Excellent control of the intermediate states was achieved in these samples by means of a proper sequence of voltage waveform.

In this work we present detailed analysis of how the conductance of the cells vary with the stimulus amplitude and time. We obtain quantitative expressions which can be used in analog application such as neuromorphic circuits or bioinspired circuits.

\section{Experimental setup}

The devices under investigation are TiN/Ti/20 nm- $\mathrm{HfO}_{2} / \mathrm{W}$ metal-insulator-metal (MIM) capacitors RRAMs. 20 nm-thick $\mathrm{HfO}_{2}$ films were deposited by atomic layer deposition (ALD) at $225{ }^{\circ} \mathrm{C}$ using tetrakis(dimethylamido)hafnium and $\mathrm{H}_{2} \mathrm{O}$ as hafnium and oxygen precursors, and $\mathrm{N}_{2}$ as carrier and purge gas. Top (TiN/Ti) and bottom (W) electrodes were grown by magnetron sputtering.

Electrical measurements were carried out putting the sample in a light-tight, electrically shielded box. DC-current and admittance were measured using a Keithley 4200SCS semiconductor analyzer. The voltage was applied to the TiN/Ti top electrode with the $\mathrm{W}$ bottom electrode grounded. The small signal admittance parameters were measured by adding a $500 \mathrm{kHz}$ small signal of $30 \mathrm{mV}$ r.m.s. to the d.c. bias voltage. A parallel admittance model was selected which directly provides the real component value of the admittance (conductance, G) and the imaginary one (susceptance, B). To prevent the effect of parasitic elements due to the experimental setup (wiring, needles, etc.) a four-probe configuration was used. At each electrode, one probe was used to apply and measure the voltage, while the other one sensed the current. A careful short/open calibration process was performed to eliminate the experimental bench parasitic artifacts. After compensation, impedance values lower than $4 \Omega$ were measured in short-circuit, and higher than $10^{9} \Omega$ in open-circuit configuration. Therefore, we can ensure that the measured values are only due to the devices under test. 


\section{Results and discussion}

In a previous work [24] we describe in detail how the bipolar switching of these $\mathrm{TiN} / \mathrm{Ti} / 20 \mathrm{~nm}-\mathrm{HfO}_{2} / \mathrm{W}$ devices were electroformed by applying a voltage of around 4 $\mathrm{V}$ to the pristine sample and using a current compliance of $50 \mathrm{~mA}$. Once the conductive filaments were formed, the low resistance state (ON state) was achieved by applying a voltage bias of $+1.2 \mathrm{~V}$, whereas the high resistance state (OFF state) was reached when applying a negative voltage of $-1.6 \mathrm{~V}$. Bipolar resistive-switching cycles showed excellent repetitiveness and a window between ON and OFF states of around $10 \mathrm{~mA}$ at $-0.1 \mathrm{~V}$. Moreover, we have found that these devices show a multilevel behavior which can be controlled in an easy and precise way. Figure 1 and 2 are examples of which we called cumulative erasing. In Fig. 1 we depart from set conditions and we apply negative voltage triangular ramps with increasing amplitude. Gradually, the reset process is accumulated from cycle to cycle. It is also important to note that when voltage returns to zero the state does not change. Only when the voltage exceeds the maximum value of the previous cycle, an increment of the reset process occurs. Similarly, a cumulative writing process can be achieved as Fig. 2 illustrates. Departing from full reset a stateto-state sequence can be obtained by increasing the voltage

amplitude from one cycle to the next one. In this case, we must point out that multilevel states in the set region without having to change the compliance current as suggested by Chang et al. [2]. A noticeable asymmetry is observed in the evolution of states in both regions. While in the set region the evolution is gradual from values of the

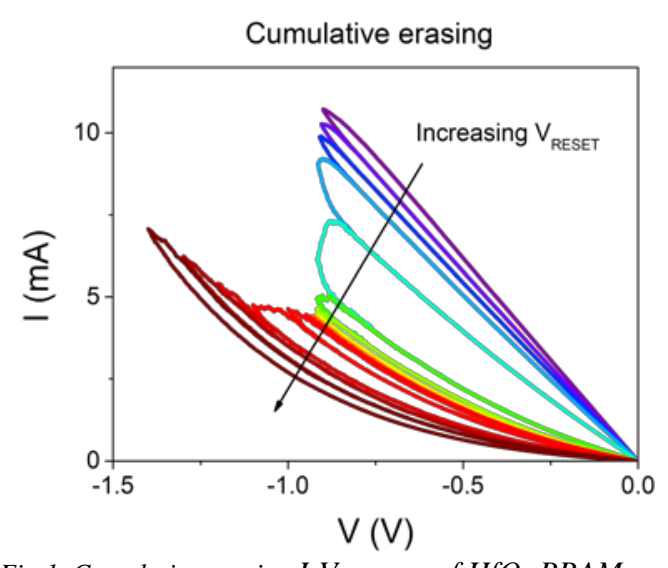

Fig.1. Cumulative erasing I-V curves of $\mathrm{HfO}_{2} \mathrm{RRAM}$ departing from full-set conditions

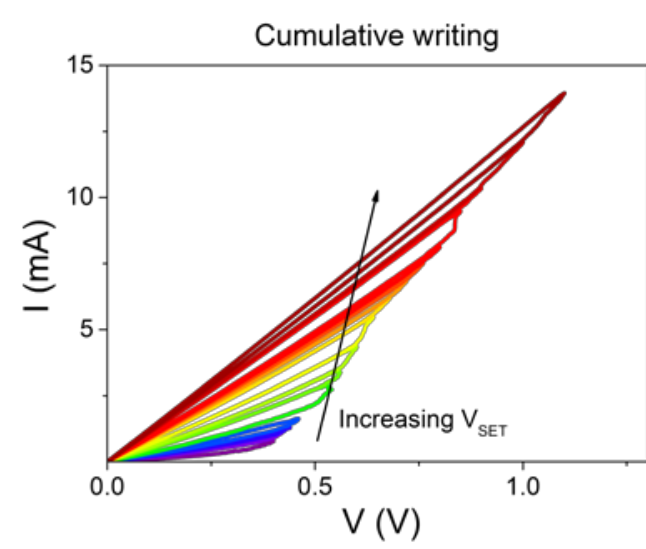

Fig.2 Cumulative writting I-V curves of $\mathrm{HfO}_{2} \mathrm{RRAM}$ departing from full-reset conditions 
set tension of approximately $0.4 \mathrm{~V}$, in the region of reset the transition is much more abrupt and starts at voltage values of about-0.9 V.

In Ref. 24 well-defined admittance loops between 0 and a positive voltage varying from +0.1 to $+1.2 \mathrm{~V}$ (ON state), or between 0 and a negative voltage varying from -0.1 to $-1.6 \mathrm{~V}$ (OFF state) were reported in the range 20 - $500 \mathrm{kHz}$. Full reset cycles departing from different initial set conditions and vice-versa were recorded, so that the initial state of each loop changed whereas the back-to-zero trajectories remained identical. Cumulative memory writing and erasing are obtained in this way. In Figs. 3 and 4 we plot the cumulative erasing and writing conductance cycles obtained at a a.c. signal frequency of $500 \mathrm{kHz}$. In these figures we have also plotted (dotted lines) the conductance vs. voltage dependency at the OFF state, Goff.

We assume that this curve is symmetric, meaning that $G_{\text {off }}$ only depends of the absolute value of the voltage. The differences between the experimentally measured values, $G$, and the off-state, $G_{\text {off, }}$ can be attributed to the term due to the conduction filaments contribution itself. Therefore, Figs. 5 and 6 represent the conductive-filament conductance during cumulative erasing and writing process, respectively. We can see that when the voltage increases in one cycle, the conductance signal follows the same trajectory as in the previous back-to-zero voltage swing, so indicating the device remember its previous state, and each new state departs from the previous one. In other

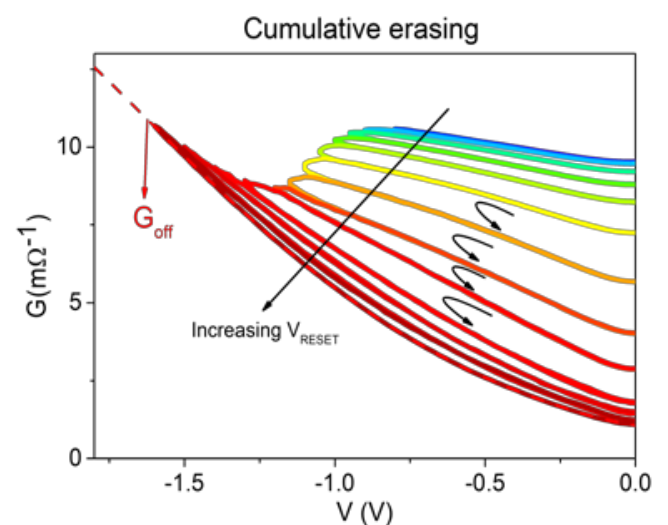

Fig.3.Experimental $G-V$ curves showing cumulative erasing of $\mathrm{HfO}_{2}$ RRAM departing from full-set conditions. Dotted line represents the conductance $G-V$ fitting on the OFF state.

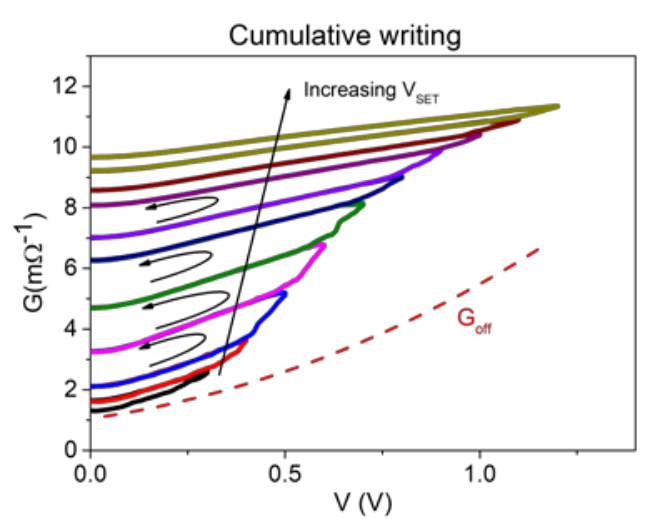

Fig.4. Experimental $G-V$ curves showing cumulative writing of $\mathrm{HfO}_{2}$ RRAM departing from full-reset conditions. Dotted line represents the conductance $G$ $V$ fitting on the OFF state. 


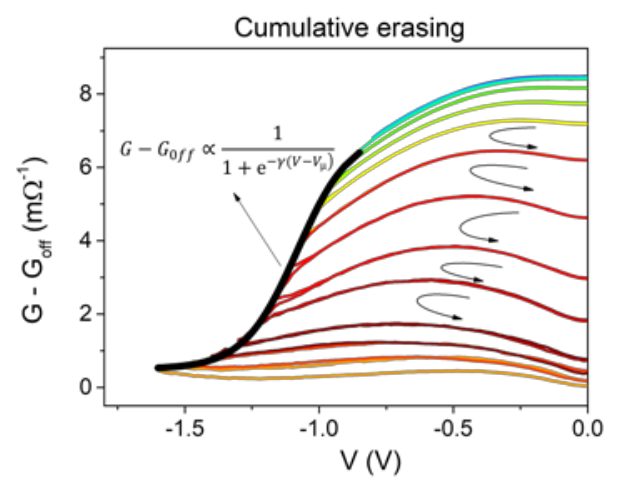

Fig.5. Cumulative erasing $\mathrm{G}-\mathrm{V}$ curves of $\mathrm{HfO}_{2}$ RRAM departing from full-set conditions after subtracting the OFF-state term $G_{\text {off. }}$ The reset process has been fitted to a sigmoidal function (thick line).

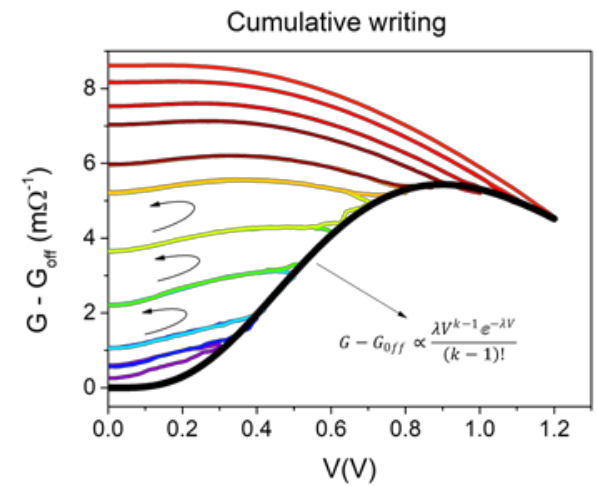

Fig.6. Cumulative writing $G-V$ curves of $\mathrm{HfO}_{2}$ RRAM departing from full-reset conditions after subtracting the OFF-state term $G_{\text {off. }}$ The set process has been fitted to an Erlang type function (thick line).

words, the maximum value of the bias voltage applied in a cycle determines the backto-zero trajectories. In short, the evolution of the state with the applied voltage is indicated in Figs. 3 and 4 by the thick lines. We note that the behavior is different for erasing than for writing. The erasing process follows a sigmoidal function:

$$
G-G_{0 f f} \propto \frac{1}{1+e^{-\gamma\left(V-V_{\mu}\right)}}
$$

with $V_{\mu}=-1.08 \mathrm{~V}$ and $\gamma=10 \mathrm{~V}^{-1}$. This expression can be interpreted in terms of the energy necessary to activate the oxygen to move to the top electrode. When the negative voltage is lower than $V_{\mu}$ the oxygen vacancies cannot be activated. More negative voltage values provide energy enough to activate the migration of the oxygen vacancies. The migration of vacancies actually consists on oxygen ions coming from the top electrode. The sigmoidal dependency indicates that the reset process is an autocatalytic process.

In contrast, cumulative writing process is better described by an Erlang function:

$$
G-G_{0 f f} \propto \frac{\lambda V^{k-1} e^{-\lambda V}}{(k-1) !}
$$

where $k=5$ is the shape parameter and $\lambda=4.5$ is the rate parameter. That means that the set process consists of the sum of $\mathrm{k}$ independent and identically distributed mechanisms, each having an exponential distribution. 
Moreover, we have also studied the time dependence of conductance. We have obtained the conductance transients for various erasing voltages after a full-set state (Fig. 7) and for various writing voltage after a full-reset state (Fig. 8). These transients are very different as well. Erasing transients are exponential and become faster as the erasing voltage increases. In contrast, the writing transients are parallel for long times and differ in the values for the very short times. That is, the set process is the result of adding several processes with very different time constants.

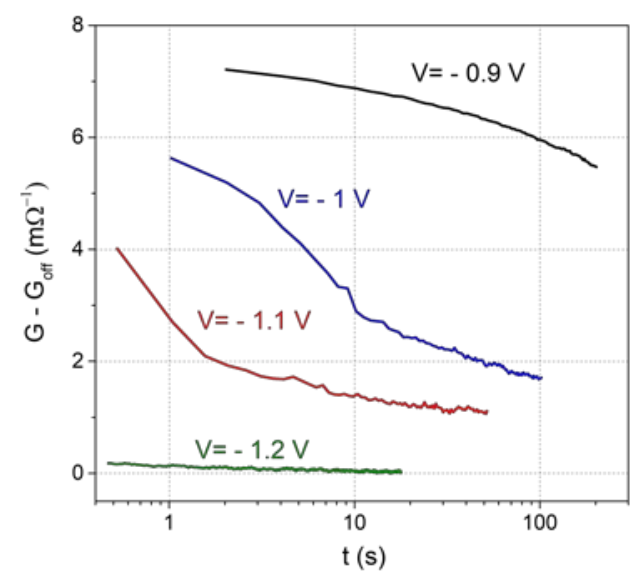

Fig. 7. Conductance transients of $\mathrm{HfO}_{2} \mathrm{RRAM}$ departing from full-set conditions after subtracting the OFF-state term $G_{\text {off }}$ at different negative voltage values

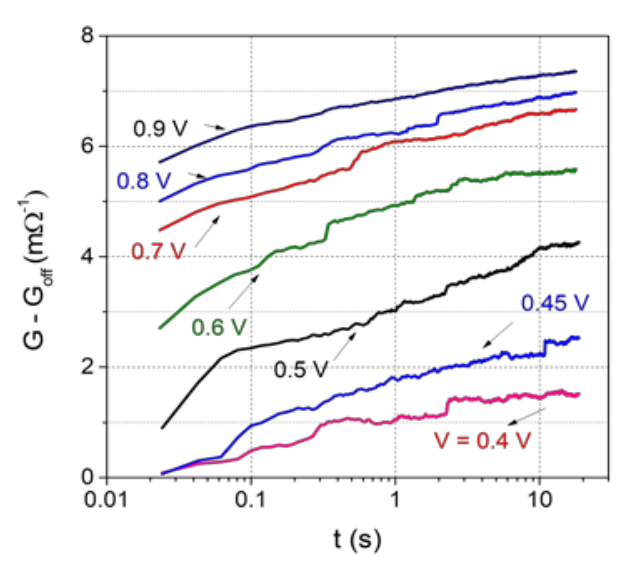

Fig. 8. Conductance transients of $\mathrm{HfO}_{2}$ RRAM departing from full-reset conditions after subtracting the OFF-state term $G_{\text {off }}$ at different positive voltage values.

These results can be interpreted at microscopic level in terms of the formation of interfacial barrier between the top electrode and the conductive filament, as proposed by Chang et al. [2]. Reset dynamics can be explained as follows: when the conductive filament is formed the applied voltage is homogeneously distributed along the entire conductive filament and moderate electric field exists everywhere. When the electric field reaches a value high enough some oxygen vacancies are activated to migrate to the top electrode and the filament is interrupted. Once the filament is partially dissolved the electric field suddenly increases at the interfacial energy barrier is formed between the top electrode and the switching layer so favoring the migration of new oxygen vacancies and causing a fast reduction of the conductivity. However, at the same time the thickness of the interface layer increases and this makes it difficult to migrate new vacancies. That causes that the time required to emit a new oxygen vacancy exponentially increases. 
In the case of the cumulative writing, as the conductive filament is partially interrupted at the reset state, the electric field at the interface barrier is high even at low values of the applied voltages. Therefore, the minimum electric field needed to move vacancies from the top electrode to the filament is achieved at lower values than for the erasing process and conductance increases at voltages as low as $0.35 \mathrm{~V}$. The higher the voltage the higher the electric field and, consequently, the greater the number of oxygen vacancies incoming to the switching layer and the velocity of the conductivity increasing. As the interface is filled with vacancies, its thickness diminishes, which favors incorporation of new vacancies and, then, the writing process does not saturate. But, at the same time, the increase of the conductivity causes an increase of the ohmic fall in the conductive filament and, therefore, a decrease of the electric field at the interface which slows down the writing process to long times.

\section{Conclusion}

In this work, we have proved that the dynamics of set and reset processes on resistive switching memories depends on the voltage applied and time. These devices show a multilevel behavior which can be observed controlled and accurately controlled by measuring the d.c. current or a.c. conductance when applying adequate voltage waveforms. The study of the conductance signal of $\mathrm{HfO}_{2} \mathrm{RRAM}$ memory cells indicates that mechanisms responsible for the set and reset processes are very different. Voltage dependencies indicate that the Set process is described by an Erlang distribution which is compatible with a percolation process, whereas the Reset fits to a sigmoidal function and can be related to an autocatalytic oxidation reaction. Time dependencies are very asymmetric as well. Erasing transients are exponential and become faster as the erasing voltage increases. In contrast, the writing transients are parallel for long times and differ in the values for the very short times. We propose a microscopic explanation in terms of the formation of interfacial barrier between the top electrode and the conductive filament. 


\section{Acknowledgement}

This work was funded by the Spanish Ministry of Economy and Competitiveness and the FEDER program through project TEC2017-84321-C4-2-R. This work has made use of the Spanish ICTS Network MICRONANOFABS.

Authors would like to acknowledge Prof. Campabadal group from the Institute of Microelectronics of Barcelona (IMB-CNM-CSIC, Spain) for providing the samples of this study.

\section{References}

[1] D. Ielmini, R. Waser, Resistive Switching: From fundamentals of Nanoionic redox processes to memristive device, Wiley, 2016. https://doi.org/10.1002/9783527680870.ch1

[2] K.-C. Chang, T.-C. Chang, T.-M. Tsai, R. Zhang, Y.-C. Hung, Y.-E. Syu, Y.F.Chang, M.-C. Chen, T.-J. Chu, H.-L. Chen, C.-H. Pan, C.-C. Shih, J.-C. Zheng, and S. M. Sze,Nanoscale Res. Let. 10, 120 (2015). https://doi.org/10.1186/s11671$\underline{015-0740-7}$

[3] P. Gonon, M. Mougenot, C. Vallee, C. Jorel, V. Jousseaume, H. Grampeix, and F. El Kamel, "Resistance switching in HfO2 metal-insulator-metal devices,” J. Appl. Phys. 107, 074507 (2010). https://doi.org/10.1063/1.3357283

[4] M. Lanza, G. Bersuker, M. Porti, E. Miranda, M. Nafria, and X. Aymerich, "Resistive switching in hafnium dioxide layers: Local phenomenon at grain boundaries,” Appl. Phys. Lett. 101, 193502 (2012). https://doi.org/10.1063/1.4765342

[5] J. J. Yang, M. D. Pickett, X. Li, D. A. A. Ohlberg, D. R. Stewart, and R. S. Williams, "Memristive switching mechanism for metal/oxide/metal nanodevices," Nat. Nanotechnol. 3, 429-433 (2008). https://doi.org/10.1038/nnano.2008.160

[6] S. L. Barbera, D. Vuillaume, and F. Alibart, ACS Nano, vol. 9(1), 941-949 (2015). https://doi.org/10.1021/nn506735m 
[7] S. Brivio, E. Covi, A. Serb, T. Prodromakis, M. Fanciulli, and S. Spiga, Appl. Phys. Lett.

vol.109, 133504 (2016). https://doi.org/10.1063/1.4963675

[8] E. Covi, S. Brivio, M. Fanciulli, and S. Spiga, Microelectron. Eng. vol. 147, 41-44 (2015). https://doi.org/10.1016/j.mee.2015.04.052,

[9] Y. Matveyev, K. Egorov, A. Markeev, and A. Zenkevich, J. Appl. Phys. vol. 117, 044901 (2015). https://doi.org/10.1063/1.4905792

[10] B. Gao, L. Liu, and J. Kang, Prog. In Nat. Sci.: Mat. Int. vol. 25, 47-50 (2015). https://doi.org/10.1016/j.pnsc.2015.01.005

[11] S. Poblador, M. B. González, and F. Campabadal, Microelectron. Eng. 187-188, 148-153 (2018).https://doi.org/10.1016/j.mee.2017.11.007

[12] Y.-F. Chang, B. Fowler, Y.-C. Chen, F. Zhou, C.-H. Pan, T.-C. Chang, and J. C. Lee, Sci. Rep., 21268 (2016). https://doi.org/10.1186/s11671-015-0740-7

[13] Y.-F. Chang, T.-C. Chang, and C.-Y. Chang, Journal of Applied Physics 110, 053703 (2011).https://doi.org/10.1063/1.3630119

[14] Y.-F. Chang, B. Fowler, Y.-C. Chen, Y.-T. Chen, Y. Wang, F. Xue, F. Zhou, and J. C. Lee, Journal of Applied Physics 116, 043709 (2014).https://doi.org/10.1063/1.4891244

[15] S. Kim, Y.-F. Chang, and B.-G. Park, Royal Society of Chemistry Advances 7, 17882, (2017). https://doi.org/10.1039/c6ra28477a

[16] C.-C- Hsieh, A. Roy, Y.-F. Chang, D. Shahrjerdi, and S.K. Banerjee, Appl. Phys. Lett. 109, 223501 (2016).https://doi.org/10.1063/1.4971188

[17] C. H. Cheng, P. C. Chen, Y. H. Wu, and A. Chin IEEE Electron Device Letters 32(12), 1749 - 1751 (2011). https://doi.org/10.1109/LED.2011.2168939 
[18] I. Salaoru, A. Khiat, Q. Li, R. Berdan, and T. Prodromakis, Appl. Phys. Lett. 103, 233513 (2013). https://doi.org/10.1063/1.4840316

[19] L. Quingjiang, A. Khiat, I. Salaoru, C. Papavassiliou, X. Hui, and T. Prodromakis, Sci.Rep. 4, 4522 (2014). https://doi.org/10.1038/srep04522

[20] T. Wakrim, C. Vallée, P. Gonon, C. Mannequin, and A. Sylvestre, Appl. Phys. Lett. 108, 053502 (2016).https://doi.org/10.1063/1.4941231

[21] Y.-F. Chang, B. Fowler, Y.-C. Chen, and J. C. Lee, Progress in Solid State Chemistry 44, 75 (2016).https://doi.org/10.1016/j.progsolidstchem.2016.07.001

[22] S. Dueñas, H. Castán, O. G. Ossorio, L. A. Domínguez, H. García, K. Kalam, K. Kukli, M. Ritala, and M. Leskelä, in 2017 32nd Conference on Design of Circuits and Integrated Systems (DCIS), Barcelona, Spain, 22-24 November 2017 (IEEE, 2018). Google Scholar

[23] S. Dueñas, H. Castán, H. García, E. Miranda, M. B. González, and F. Campabadal, ， Mic Eng 178, 30-33 (2017). https://doi.org/10.1016/j.mee.2017.04.020

[24] H. Castán, S. Dueñas, H. García, O. G. Ossorio, L. A. Domínguez, B. Sahelices, E. Miranda, M.B. González, and F. Campabadal, J. of App. Phys. 124, 152101 (2018).https://doi.org/10.1063/1.5024836 


\section{${ }^{*}$ Declaration of Interest Statement}

\section{Declaration of interests}

$\bigotimes$ The authors declare that they have no known competing financial interests or personal relationships that could have appeared to influence the work reported in this paper.

$\square$ The authors declare the following financial interests/personal relationships which may be considered as potential competing interests:

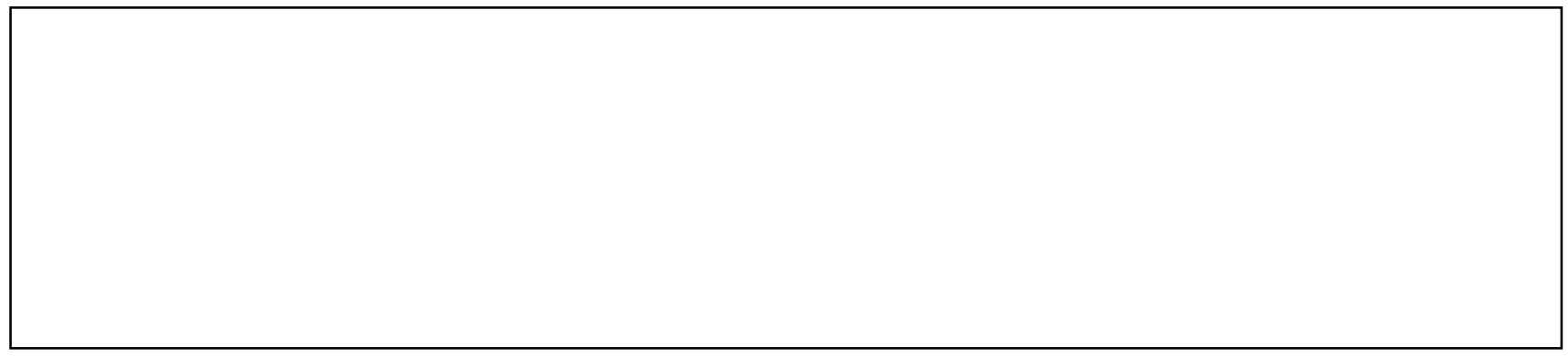


${ }^{*}$ Conflict of Interest Statement

\section{Conflict of interest Statement}

$\bigotimes$ The authors declare that this work does not have any conflict of interest 